\title{
SURGICAL TREATMENT OF NARROWING OF THE INTERNAL CAROTID ARTERY
}

\author{
BY \\ C. H. EDWARDS, F.R.C.P. NEIL S. GORDON, M.D., M.R.C.P. \\ From St. Mary's Hospital, London
}

In the past few years the frequency with which atheromatous obstructions of the internal carotid artery in the neck occur has been realized. The growth of percutaneous arteriography has served to remind us of the neglected pathological studies of Hultguist (1942) and others, but even now the arterial bridge between thorax and cranium is seldom studied at necropsy. Hutchinson and Yates (1957) have shown that obstructing atheroma commonly occurs in this arterial bridge whilst the vessels above and below are relatively or wholly healthy. The variable clinical picture of stenosis of the internal carotid artery in the neck has been described by Johnson and Walker (1951), Fairburn (1957), and Symonds (1957). Advances in technical ancillaries to surgery allowed the first such case to be operated upon successfully at St. Mary's Hospital (Eastcott, Pickering, and Rob, 1954). Later a group of cases were reported by Rob and Wheeler (1957), and these were augmented and described in greater detail by Edwards, Gordon, and Rob (1960). This paper is composed of a further follow-up study of a still larger number of patients.

Before describing the results of this follow-up, however, it might be useful to summarize briefly what is known about this disease. Hunt (1914) was the first to recognize its clinical importance, and subsequent authors have agreed that atheroma is much the commonest cause of either a partial (stenosis) or complete (occlusion) obstruction to blood flow within the carotid arteries. The usual site of such a lesion is at a centimetre or so distal to the origin of the internal carotid artery from the common trunk. If a stenosis becomes an occlusion it usually does so by a thrombosis which often spreads upward to the point of origin of the first branch, the ophthalmic artery. The block, for technical reasons, is then no longer amenable to surgical removal. The internal carotid artery supplies the ipsilateral eye and the major part of the ipsilateral cerebral hemisphere. The collateral circulation to the latter is by the circle of Willis and its tributaries, while the eye receives a more efficient collateral circulation from the external carotid artery. This anatomy makes it easy to understand that the clinical picture of a block of the internal carotid artery in the neck is variable.

An occlusion is compatible with no symptoms or signs; a stenosis may cause a complete contralateral hemiplegia. On occasion the ipsilateral eye may be blinded in addition to the hemiplegia. Sometimes the ocular symptoms occur alone. The clinical picture, of variable extent or degree, may present transiently or repetitively (here called "stuttering"), gradually and permanently (here called "gradual"), or suddenly and permanently (here called "sudden"), and, of course, transiency may later lead to permanency. There is one clinical picture that is diagnostic, but this is relatively rare; it is an ipsilateral blindness together with a contralateral hemiplegia which may come on in a stuttering, gradual, or sudden fashion. In any patient hemihypaesthesia, dysphasia, and hemianopia may be added to the hemiplegia. A systolic bruit may sometimes be heard over the arterial stenosis but not over an occlusion.

\section{Case Material}

When the second follow-up examination was done at St. Mary's Hospital 97 patients had been admitted in a period of six and a half years. There were 81 men and 16 women, their ages varying between 28 and 76 years, with an average of 59 . To avoid repetition the first 40 patients, already reported in detail (Edwards et al., 1960), are referred to as group $A$ and the remaining 57 as group B. A complete arterial block is referred to as an "occlusion," a partial block as "stenosis."

Lesions of the artery were shown on the left side in 50 patients, on the right side in 29 , and on both sides in 18. However, bilateral arteriograms were not always taken, so that the frequency of bilateral lesions is not known. The onset of symptoms is considered under three headings ; stuttering, sudden, and gradual (Table I).

\begin{tabular}{ll|c|c|c|c}
\multicolumn{8}{c}{ TABLE I.-Mode of Onset of Symptoms } \\
\hline & & Group A & Group B & Total \\
\hline Stuttering &.. &. & 19 & 35 & 54 \\
Sudden &. &. & 14 & 16 & 30 \\
Gradual &.. &.. & 7 & 6 & 13 \\
\hline
\end{tabular}

It will be seen that the majority presented with a history of transient symptoms and that the proportion of these was greater in group B. The difference may reflect only an improving selection for operation. The symptoms of the patients in the two groups showed no significant difference. Weakness was the commonest symptom, occurring in 84 . The arm was affected more often than either the face or the leg, suggesting that the anoxia was mostly within the distribution of the middle cerebral artery. Only 39 patients complained of sensory symptoms, usually transient and with the 
same distribution. All but five of these had motor symptoms as well. Twelve patients gave a history of impairment or loss of consciousness at the start of the illness and 10 had epileptic seizures, but in only two were these of a focal nature. A history of difficulty in speaking was common, as might be expected from the preponderance of left-sided arterial lesions, but it was sometimes difficult to be certain whether the symptoms indicated a dysphasia or a dysarthria. Fifty-seven patients complained of their speech, in 27 the disorder was only transient.

Among the most important symptoms are the ocular ones because of their help in reaching early diagnosis (Gordon, 1959). Sixteen patients gave a history of transient blindness of the appropriate eye, while three others became permanently blind, having originally had transient symptoms. Seven patients had a permanent defect of vision in one eye, although in one of these the affected eye was on the side opposite to the arterial lesion. The history of four patients was consistent with a hemianopic defect and five complained only of blurred vision, which was usually transient. Double vision was an occasional symptom.

Twenty-four patients had headaches, sometimes severe. In only seven were they unilateral, all but two on the side of the diseased artery. Symptoms of dementia were given by 19 patients. Faintness and postural vertigo were infrequent complaints, but one patient presented with giddiness on pressure over a swelling in the neck which turned out to be a fibrous thickening overlying an atheromatous plaque of the internal carotid artery. Another patient gave a history of brief attacks of left-sided weakness brought on by bending the neck, and when the artery was explored it was found to be surrounded by fibrous adhesions although the lumen was normal. In one patient, who had fallen and struck the side of the neck, the arterial lesion was secondary to this injury. After a brief interval consciousness was lost and on recovery a severe hemiplegia had developed. The internal carotid artery was later found to be thrombosed throughout its course in the neck. Symptoms of arterial disease in other parts of the body, such as angina pectoris or intermittent claudication, were common. The history prior to admission varied from one day to five years. It was usually short, being less that six months in 60 patients and less than a year in a further 15 .

The abnormal physical signs in the two groups were in fair agreement except that in group B greater attention had been paid to the local examination of the arteries of the neck. The arterial pulse was noted to be diminished on the affected side in about the same proportion in the two groups, but a bruit was heard over the artery in 18 patients in group B and in only one patient in group $\mathrm{A}$.

Although no formal tests of intellect were made dementia was noted in 26 patients. Twenty-six patients had dysphasia, predominantly expressive, and 11 others had a slight dysarthria.

Retinal changes were infrequent, only two patients showing a hypertensive retinopathy and nine arteriosclerotic changes of more than mild degree. Optic atrophy was seen in four patients, all of them having a history of a year or more. The visual acuity of the ipsilateral eye was diminished in 10 patients while nine showed a hemianopic field defect appropriate to the side of the arterial lesion.
Sixty-eight patients had a hemiparesis, one of them a double hemiparesis. Twenty-eight had sensory signs in addition and in two further cases the sensory signs occurred alone. The arm was involved more than any other part of the body. In five patients reflex abnormalities were the only evidence of an uppermotor-neurone lesion, and in 10 bilateral reflex changes were the only evidence of such a lesion on both sides. Thirty-eight patients had a systolic blood-pressure of over $150 \mathrm{~mm}$. $\mathrm{Hg}$ and 18 of over $200 \mathrm{~mm}$. Hg. Forty had a diastolic pressure of over $90 \mathrm{~mm}$. $\mathrm{Hg}$ and 9 of over $120 \mathrm{~mm}$. Hg. A raised blood-pressure per se does not appear to contraindicate operation.

The investigations that need to be considered are carotid arteriography and electroencephalography. Ninety-one patients were subjected to the former test (Table II).

TABLE II.-Carotid Arteriography in 91 Patients with Carotid Artery Disease (7 Patients Had Complete Block on One Side and Partial Stenosis on the Other)

\begin{tabular}{|c|c|c|c|c|}
\hline & & Group A & Group B & Total \\
\hline 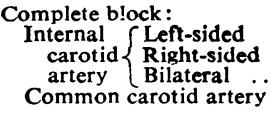 & $\begin{array}{l}. . \\
\because \\
\cdots\end{array}$ & $\begin{array}{c}13 \\
5 \\
1 \\
1(L)\end{array}$ & $\begin{array}{l}4 \\
6 \\
1 \\
1 \text { (R) }\end{array}$ & $\begin{array}{r}17 \\
11 \\
2 \\
2\end{array}$ \\
\hline Total & .. & 20 & 12 & 32 \\
\hline$\underset{\text { stenosis }}{\text { Partial }}\left\{\begin{array}{l}\text { Left-sided } \\
\text { Right-sided } \\
\text { Bilateral }\end{array}\right.$ & $\begin{array}{l}. \\
\because \\
\because\end{array}$ & $\begin{array}{r}12 \\
7 \\
1\end{array}$ & $\begin{array}{r}26 \\
12 \\
8\end{array}$ & $\begin{array}{r}38 \\
19 \\
9\end{array}$ \\
\hline Total & & 20 & 46 & 66 \\
\hline
\end{tabular}

One patient, already mentioned, who was found to have adhesions in the region of the carotid bifurcation on the right side had a normal common carotid arteriogram on both sides. The fewer examples of complete carotid occlusion shown on arteriography in group B may reflect a better selection of patients for surgical treatment. Another difference between the two groups was the greater number of bilateral arteriograms done in group B. Twelve patients in group A and 24 in group B were examined in this way-two showed a bilateral occlusion, six an occlusion of one side and a normal artery on the other side, seven an occlusion on one side and a stenosis on the other, eight a stenosis on one side and a normal artery on the other, and 10 a stenosis on both sides. Two patients showed a stenosis at the usual site, but one of them had a second stenosis on the opposite side at the level of the junction between first and second cervical vertebrae, and the other had a second stenosis, again on the opposite side, within the cavernous sinus. Bilateral arteriograms were normal in one patient with adhesions around the carotid artery. One patient showed an occlusion of both the internal and the external carotid arteries on the same side and another patient had a stenosis of these two arteries on the same side. Among the seven patients with an occlusion on one side and a stenosis on the other, three showed bilateral signs and two showed signs confined to the side opposite to the partial occlusion.

Six patients were operated on without previous arteriography. Two of these had a well-marked bruit over the affected artery and one was the patient who was found to have a fibrous swelling around an atheromatous plaque. One emergency operation was done, after attempted arteriography, because of a haematoma around the artery.

An electroencephalogram was carried out on 54 patients. It was normal in 22 and showed only diffuse 
abnormalities of non-specific type in three. The records of the remaining 29 showed focal abnormalities on the appropriate side although in two of them only on compression over the affected artery. The abnormalities consisted of irregular slow-wave activity within the theta and delta range tending to be most marked over the temporal region.

\section{Surgical Treatment}

Eighty-two patients were operated upon. A stenosis was removed from the appropriate artery in 61 . In two of these there was an occlusion of the internal carotid artery on the contralateral side although this did not appear to be causing symptoms. In four others a partial obstruction was removed from both sides, although in only one of these patients was there evidence of bilateral cerebral disease, and then only in the form of reflex abnormalities. One patient was operated on twice owing to a recurrence of a stenosis which again produced symptoms and signs. A stenosis of the internal carotid artery had been demonstrated radiologically in 56 of these 61 patients. In one of the infarction, and one because death occurred within three days of admission to hospital. Another of these patients showed no abnormal signs on examination and another no abnormality apart from a mild dementia. The arteriogram of this patient showed only a slight stenosis of the internal carotid artery in the neck, and this may not have been the cause of the illness. The sixth patient was suffering from widespread atheroma.

\section{Assessment of Patient at Follow-up Examination}

The second follow-up examination and assessment was made three years after the first (Edwards et al., 1960). The interval between operation, or discharge from hospital if none had been performed, and the follow-up examination varied between six and a half years and one month, but the period was less than a year in only eight cases. Sixty-six patients were examined personally-three by their own doctor-five had died, and three were untraceable. The present state of the 69 patients was assessed by mutual agreement between ourselves (Table III). Nineteen had improved

TABLE III.-Assessment of 69 Patients Included in Follow-up Examination

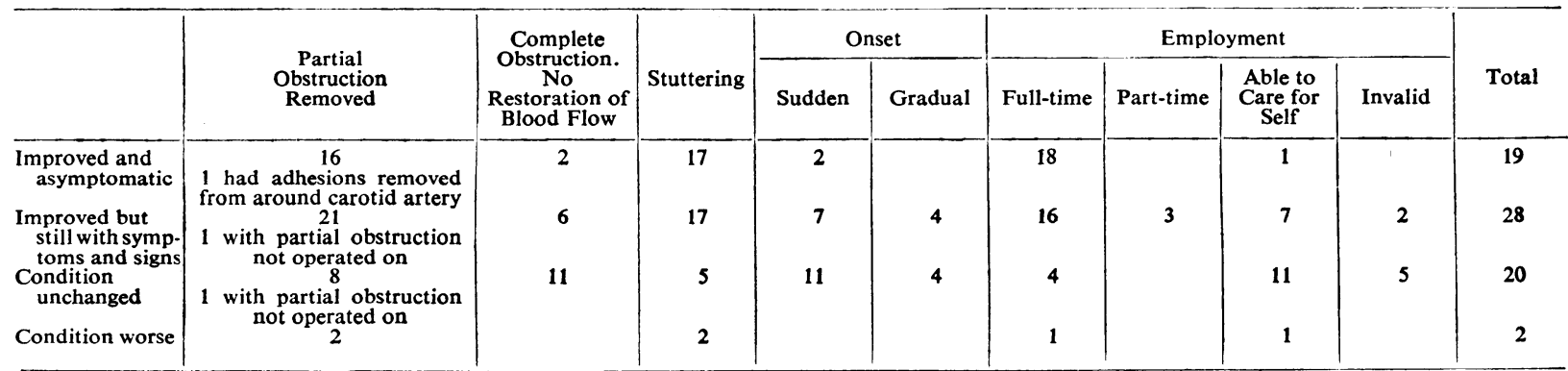

patients in group $\mathbf{A}$ the stenosis was on the side of the hemiplegia, the other internal carotid artery being completely blocked and the operation being advised in an effort to increase the total blood flow to the brain. As has already been reported (Edwards et al., 1960) two of the patients in group A were operated on shortly after the onset of a complete occlusion of the internal carotid artery but without benefit. No such operations were done in group B. One of the patients in group B had adhesions removed from around the carotid bifurcation, but the artery was not opened. Thromboendarterectomy was performed in all but six patients, a resection and end-to-end anastomosis being carried out in four and homografts being used in two. Details of the surgical technique have previously been given (Edwards et al., 1960).

It was not possible to re-establish the blood flow in 18 patients; only four of these were in group B, suggesting an improved selection for operation. It was decided for various reasons not to operate on 15 patients. Eight of these were in group $\mathbf{A}$ and seven in group $\mathbf{B}$. Two had a complete occlusion of both internal carotid arteries. Three had an occlusion on one side and a stenosis on the other side, and one of these showed evidence of brain-stem ischaemia and two showed signs of bilateral cerebral damage. Four patients had an occlusion on one side and two of these had a history of a month or more, making the operation doomed to failure. One had signs of a bilateral upper-motorneurone lesion and one dysphasia only, which was already improving on admission to hospital. Six patients with stenosis were not operated upon; one because of advanced renal disease, one because of a recent cardiac and were free from all symptoms and signs, and a further 28 had improved when their condition was compared with that on admission to hospital. Although symptoms and signs were still present in this group they were usually mild. The condition of 20 patients had remained unaltered and two had become worse.

Of the 19 patients free of symptoms and signs only three had shown any significant neurological abnormality before operation. Sixteen of them had a stenosis of the internal carotid artery suscessfully removed and one was found at operation to have dense adhesions around the artery though no obstruction within its lumen. An occlusion of the artery had been demonstrated in the other two patients and it had not been found possible to re-establish the blood flow. The onset of symptoms in all but two patients in this group of 19 was "stuttering," the exceptions having suddenly developed a slight hemiplegia. All except two had returned to their previous occupations. One of the three with neurological signs before operation had obtained labouring work, having once been a bookbinder, and a woman of 70 had ceased to do any of her housework. She had also shown evidence of a hemiplegia before operation.

The disability of the 28 patients who had improved but still had symptoms and signs varied but was mostly mild. Nineteen had returned to work-14 to their previous employment, two to lighter jobs, and three to part-time work. Sixteen of these had had a stenosis removed from an internal carotid artery and three were found to have an occlusion. Seven patients were unable to work although they could care for themselves. Two of these had an occlusion of one artery and five a 
stenosis, in one case of both internal carotid arteries and in another associated with severe atheroma of the artery in the cavernous sinus. Two patients, although slightly improved, were still invalids. One had an occlusion of one artery and the other had been operated on for the removal of a stenosis from both internal carotid arteries.

The condition of 20 patients was unchanged. Four of them had been able to continue with their previous work. Of these, two had an occlusion and two a stenosis of one artery. Of the 11 patients who had been unable to return to work, six had an occlusion of one artery. One of the patients with a stenosis had evidence of generalized cerebrovascular disease. The remaining five patients in this group were complete invalids, although two only had a stenosis of the appropriate carotid artery.

The condition of two patients was worse after operation. One had had a stenosis removed, but soon afterwards the arm became weaker and had remained so ever since. The other patient's condition also deteriorated after such an operation, and a subsequent arteriogram showed a completely occluded artery.

Twenty-five patients were known to have died within the follow-up period-16 in group A and 9 in group B. In only one case could death be directly attributed to the operation-removal of a stenosis. When the patient's condition deteriorated the next day a reexploration of the neck showed that the artery had completely thrombosed, and necropsy revealed softening of the brain on both sides. Two other patients died from pulmonary embolism soon after operation. Five patients died from cerebral haemorrhage while still on anticoagulant therapy. Post-mortem examination showed that the disobliterated artery had remained patent in them all. One other such patient almost certainly died of cerebral haemorrhage, but permission for a necropsy was refused.

In many cases no detailed account of the cause of death could be obtained. Two patients died of coronary thrombosis, one from myocardial degeneration, one from left ventricular failure, two from bronchopneumonia, one from renal disease, and one after amputation of a gangrenous leg. In 5 cases death was said to be due to cerebral thrombosis, but in only two was this confirmed at necropsy. One of these showed a massive infarction of the left temporal lobe and the other infarction of the left parietal lobe as well as thrombosis of the upper end of the left vertebral artery. In three cases no accurate information could be obtained.

As in the previous follow-up (Edwards et al., 1960) there is no doubt that the best results are in those patients with a "stuttering" type of onset, few if any permanent symptoms, and a partial arterial block. Twenty-seven such patients had been operated upon and had improved, 14 being free of all symptoms and signs. Patients with a sudden onset of symptoms do not have such a good prognosis, only 7 out of 13 operated on for stenosis having improved and only two of these being free of all symptoms and signs.

Among the 69 patients who were re-examined 42 had returned to work, 20 were able to care for themselves, and 7 were complete invalids. In the first group there were 29 with a "stuttering" onset of symptoms, and 34 had a stenosis of the internal carotid artery; this had been removed in 32 cases, confirming the good prognosis in this type of patient.

Finally, a brief analysis may be made of the 31 patients in group $A$ alive at the time of the second examination. In 22 patients the clinical findings were thought to be the same and there had been a slight improvement in two others. In none of these had there been any change in their ability to work or care for themselves. A further seven had died. These findings after an interval of three years would suggest that the disability that may result from carotid thrombosis will show little change after any initial improvement that may occur.

\section{Discussion}

The extreme variability of the clinical picture in extent and in degree has been stated, but when selesting the most suitable cases for operation the most important Iariable is the duration of symptoms. This indicates the prognosis for surgery more accurately than the degree of disability, the follow-up examination having shown clearly that the patient with intermittent symptoms is likely to benefit most from removal of a carotid stenosis. The causes of this variability in duration are not understood, and a concept of a more dynamic intracranial circulation than is usually considered may be necessary to account for these vagaries (Brain, 1957). An adequate collateral circulation can explain the asymptomatic arterial occlusion, but one seldom appreciates the variable factors that must be present to account for intermittent symptoms accompanying an arterial block. Experimentally it can be demonstrated that areas of brain above such a block are more than usually vulnerable to arterial hypotension, but there are probably other natural variables.

At the moment it is clear that angiography is essential for the accurate diagnosis that surgery demands. Only the classical clinical picture previously mentioned is diagnostic, and this is rare and can happen both with an operable stenosis and an inoperable occlusion with thrombosis. The arterial bruit may mislead, being propagated from afar or from the neighbouring external carotid artery. Ophthalmodynamometry is not accurate enough to be relied upon alone but may well be useful as a simple, painless, and rapid screening process before arteriography (Liversedge and Smith, 1961 ; Lowe and Stephens, 1961). There is a growing need in many patients to assess the capacity of more than one of the arteries to the brain, and in expert hands cerebral angiography carries only a minimal risk.

Further experience confirms that only a minute number of total arterial blocks are removable. The reason is that thrombosis completes a gradual stenosis and the clot quickly extends to involve the artery within the skull and also becomes welded to the artery wall. Arteriography alone makes this essential distinction between occlusion and stenosis.

This second follow-up confirms the previously recorded surgical results (Edwards et al., 1960) and in particular that this operation is a prophylactic one. It probably does nothing to remove permanent symptoms, the results being no better than could reasonably be expected from the natural history of the disease. The best results are seen when the operation abruptly and permanently stops frequently recurring symptoms, as it almost always does. On the 
contrary, the untreated patient may become severely disabled.

It is more difficult to assess the outcome of an operation for a single transient attack, as it is not uncommon for this to be unique in the natural history of the untreated case. This summarizes the results of operating upon what might be called the appropriate artery to a particular "stroke." There is, however, a growing tendency to consider the state of the quartet of arteries to the brain and to regard them not only as supplying blood to their own particular territory but also as contributing to the circle of Willis for general distribution. This concept is the reason for considering a bilateral internal carotid artery disobliteration or an operation contralateral to an inoperable complete block. It also raises the possibility of internal carotid artery surgery to improve oxygenation of the brain-stem.

As yet few operations on the vertebral arteries have been done. There is a steady improvement of surgical technique; by-pass manœuvres, for instance, may replace the need for hypothermia. Until atheroma is curable or, more likely, preventable, surgery may stay as the most certain method of increasing the circulation to the brain.

\section{Summary}

The symptoms and signs of 97 patients known to have a lesion of the carotid arteries are analysed.

Details of the investigations and treatment are given. In 61 of the 83 operated upon it was possible to re-establish blood flow in the appropriate artery.

The results of the follow-up examination of all but three of the 72 surviving patients are given.

The following points are briefly made: (1) Atheroma is almost the sole cause of narrowing of the internal carotid artery. which may be partial or complete. (2) The clinical picture varies widely in degree, extent, and duration. These variations bear no constant relationship to the degree of arterial obstruction. The commonest clinical picture is of hemiparesis, which may occur as a single episode or in the form of transient attacks. The addition of contralateral uniocular blindness is almost pathognomonic of insufficiency of the common or internal carotid artery, but is a relatively rare presentation. (3) A complete arterial block is usually associated with a thrombus that has spread intracranially and has adhered to the vessel wall so as to become inoperable. (4) The ideal patient for surgical treatment is one who has suffered from transient and reversible symptoms due to a partial block of an internal carotid artery. In other words, the operation should be regarded as prophylactic and not curative. (5) At present pre-operative arteriography is essential.

There is a growing tendency to study, clinically and arteriographically, all the arteries carrying blood to the brain through the neck irrespective of the presumed site of ischaemia-indeed, to consider improving the blood supply to the circle of Willis rather than to any one part of the brain.

All the operations in this series were done by Professor Charles Rob or by members of the surgical unit at St. Mary`s Hospital, London. Our thanks are due to many colleagues who have sent patients from a wide area of the country. One of us (N. G.) wishes to thank the Manchester Regional Hospital Board for a grant of study leave to take part in this work.

\section{REFERENCES}

Brain. R. (1957). Lancet, 2, 857

Eastcott, H. H. G., Pickering, G. W., and Rob, C. G. (1954) Ibid., 2, 994.

Edwards, C. H., Gordon, N. S., and Rob, C. G. (1960). Quart. J. Med., 29, 67.

Fairburn, B. (1957). Brit. med. J., 2, 750

Gordon, N. (1959). Brit. J. Ophthal., 43, 257.

Hultguist, G. T. (1942). Uber Thrombese und Embolie der Arteria Carotis. Stockholm.

Hunt, J. R. (1914). Amer. J. med. Sci., 147, 704.

Hutchinson, E. C., and Yates, P. O. (1957). Lancet, 1, 2

Johnson, H. C., and Walker, A. E. (1951). J. Neurosurg., 8, 631

Liversedge, L. A., and Smith, V. H. (1961). Brain, 84, 274.

Lowe, R. D., and Stephens, N. L. (1961). Lancet, 1, 1241.

Rob, C. G., and Wheeler. E. B. (1957). Brit. med. J., 2, 264

Symonds, C. P. (1957). In Modern Trends in Neurology (2nd ser.), edited by D. Williams. Butterworth, London.

\section{RELATIONSHIP BETWEEN ARTERIAL DISEASE IN DIFFERENT SITES}

\section{A STUDY OF THE AORTA AND CORONARY, CAROTID, AND ILIAC ARTERIES}

BY

\section{J. R. A. MITCHELL, M.B., B.Sc., M.R.C.P.}

AND

\section{J. SCHWARTZ,* M.D.}

Department of the Regius Professor of Medicine, Radcliffe Infirmary, Oxford

Clinical studies have suggested that patients with arterial disease in one part of the body are likely to show or develop manifestations of disease in other arteries (McDonald, 1953 ; Richards, 1957 ; Singer and Rob, 1960 ; Juergens et al., 1960 ; Bloor, 1961). This concept of generalized arterial disease has influenced terminology, for patients with cardiac infarction are often described as "atherosclerotic individuals," and studies of patients with infarction or angina pectoris are assumed to be studies of "atherosclerosis" (Report, 1956). Necropsy surveys have, however, suggested that the severity of arterial disease in one site may not correlate with the amount of disease in other arteries (Duguid and Robertson, 1955; Robertson, 1959).

Because of this conflict between the clinical observations and the pathological findings, we have studied the prevalence, severity, distribution, and type of arterial plaques in an unselected necropsy sample and a selected group of patients with myocardial infarction.

\section{Method A}

During the first period of the study every tenth patient aged 35 years and over on whom a necropsy was performed at the Radcliffe Infirmary, Oxford, was included in our series, giving an unselected necropsy sample of 137 patients (75 males, 62 females). The age-and-sex distribution of these patients is given in Table I. In this unselected group were 15 patients (11 males, 4 females) with large areas of cardiac necrosis or replacement fibrosis (myocardial infarction-Mitchell and Schwartz, 1962). To obtain more information on the vascular state of such patients we collected a consecutive series of a further 64 patients (46 male, 18 female) with large cardiac lesions. These patients,

*Now at Division of Histopathology, Institute of Medical and Veterinary Science, Adelaide, South Australia. 